\title{
关于制訂科学工作远景 計划的意見
}

\section{發展我國的計算数学}

\author{
关肇 直
}

（中國科学院数学研究所）

近年來由于我國开始了社会主义建設, 各方面对計算数学的需要巳經逐渐顯示出來。 計算数学的發展润題已被提到日程上隶。

数学的研究对像之一就是現实世界的量的关系。在实用上, 不但要知道这利关系, 而且还要把表现这利量的关系的一些数字求出隶。这就是境, 要把所提出的問題的解答 獲得数倠形式, 这就是計算数学的任务。举一个極淺易的例, 圆周与沿徑成正比这一量 的关系的發現, 当然是一作重大的事情, 但在实用上, 我們还必須計算出这个比值秝, 至 少計算到在各种用途上足够准确的数值。又例如由于很多自然琴像的量的侧面要用微分 方程描述, 微分方程的数值解法是在实用上非常需要的。在这样的意义下, 計算数学在 整个数学中是緭有相本性的。同时, 由于在实用上碰到的計算間題范園很庶, 計算数学的 范图也是很废的。数值插值法、数值微分与皘分和与此相关的美分法等是計算数学中較 早的部阿, 例如我國早在公元 6-7 世紀就先后發現了等間距与不等間距的括值公式。在 近代, 則高等分析叮的近似方法, 包括微分方程的数值解法、積分方程的数值解法、保 鿇映像的近似方法等等, 成为数值計算中的最重要部分。但近似算法中, 各利方程最后 往往化成有笨多个未知数的代数方程組, 从而代数中的計算方法, 特別包括代数方程解 法、一狄代数方程組的解法、方陣的求逆、求方陣的固有值等等成为很多人研究的問題。

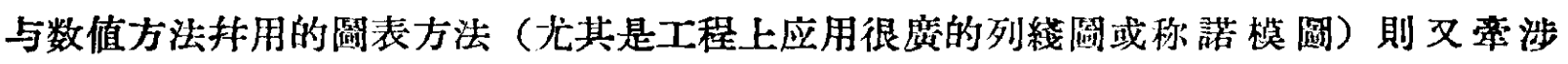
到几何学。数值計算接触的范園既然如此之廣, 于是在一定意义下可以䚺全部数学都成 为計算数学的谁备与基礎, 秚为它提供了各种工具。例如保角映像理諭本是复变数函数 睮的一部分, 敉且發展得相当好了, 但在使用这个理諭解扶流体力学、彈性力学等提出 的应用問題时, 仍有很多困难需要克服。函数論为計算数学准备了理論基礎, 而具体可 行的方法还要計算数学來提供。同样与計算数学有密切关系的, 还有綫性代数、微分方 程論、崡数逼近論等等。下面將再詳細談及这一問題。計算数学也可以誈是数学中其它

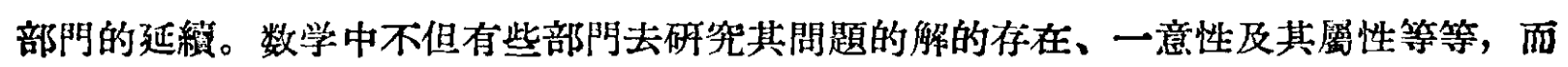

3 月号

科学通报 
且有部算数学这一分支來研究求这解的数值的具体步驟。

有些人覚得計算数学只研究一些近似算法, 不像其他数学部門那条精确, 似乎是一 阿低級的数学。这是韭常錯謎的, 正是偏重理論、輕視实用的有害覌点的反陕。計算数学 不僅要研究解某些問題的近似方法, 而且要研究这些近似方法所求泊的解的谁确程度, 即誤差必須估計出來。在这个意义下計算数学是很精确的科学。維然有些近似方沙是由 一些实用工作者在不啒謹的形式下提出的, 計算数学的任务还要研究这方江倠朋的条件

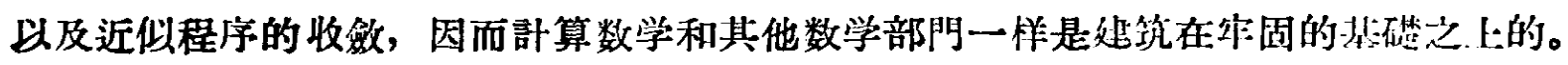

計算数学中的基碟部分自从 19 世紀以來已形成了数学中一个巨大的独立分文。在近 若干年本, 由于工業發展的需要, 对于計算数学提出了很多要求。例如在修处大䛵电站 时堤壩的强性应力、水在堤壩下的渗透的計算、飛机在飛行时所經受的阻力的計算、造 船的計算、利用数理統計于啇品检驗方面的計算等等。又在國防方面，也往往提油繁重 的計算問題。近代各國都紛紛注意郡算数学, 是有其客覌需要的。随着我國的跴会主义 工業化、國防現代化, 計算数学的發展对我們也非常迫切。由于很多实际計算繁难元長,

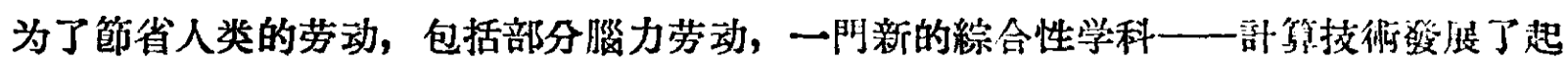
來。計算的机器化是近代科学的巨大成就。但值得注意的是計算机的發得, 特別是快速 电子訃算机的發展, 妌非如某些人想像的那样把計算数学这門学科浮娍拈。而相反的,

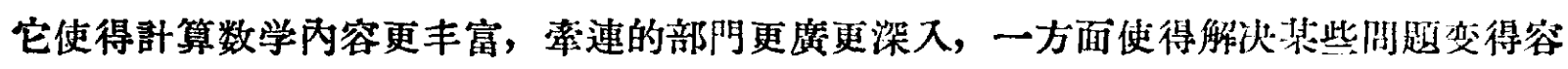
易, 另一方面也給数学提出了新的課題。因此, 如果鿁大型快速电子計算机的䈫展怕給 或正在給整个数学率來草命性的变化，我想絕不是过甚之詞。

在我國过去基礎極美的情形下急于把計算数学这一学科建立和没展起來, 垱然利不 簡單。但在世界上其他國家, 計算数学的突出淮展也是較近的事。在苏联以及在美國,

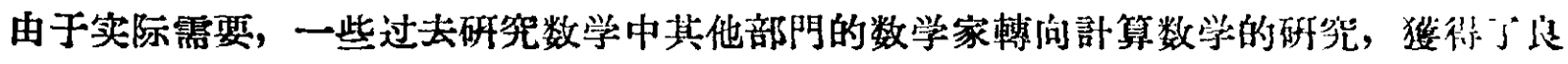

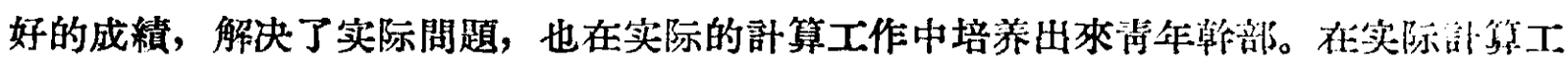
作中碰到一些間題, 又可提高到理論上去研究, 从而也丰宫数学的各部阴。侧如苏联科

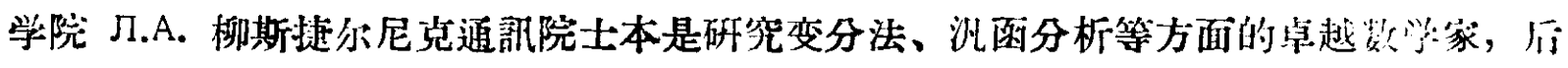

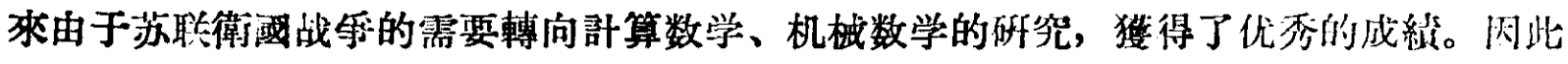
在数学各部門有成績的数学家注意計算数学是值得欢迎的。这里的另一点國际緅驗》是 培养清年必須使他們接触实际計算問題, 因此我們必須与生產部阿取得联系, 接受他們

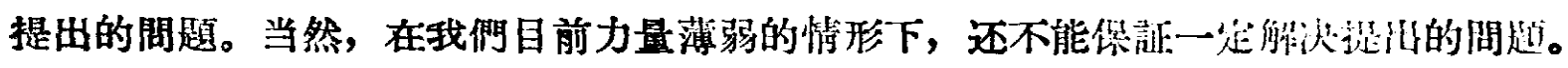
但我們决不能等幹部培养好了再去接受实际間題，而应当在船决实际阙題中去培养㲦部。

1日于計算数学在整个数学中的地位已如上退, 計算数学不可能和数学中其它部阿分

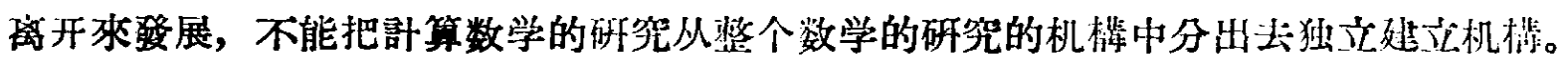
但仿效苏联科学院建立一个接受实际即算任务的服务机構“計算中心”則是很好的办法。 当然研究工作和服务工作必須祭密地結合, 研究人員应同时担任解决实际闰题方面的倾

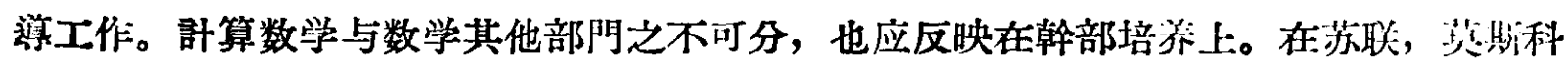
大学数学力学系設有計算数学尃業, 但这等業的課程与数学軗業的課程大体机同, 只是 少几門一般課（如几何基礎、数論、教学法)，而多近似方法、計算机概姴、山工原理一宁

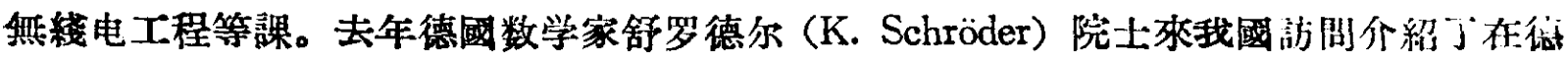


国柏林洪包特大学講授实用分析培养应用数学幹部的經驗, 也强調在大学的前丽三年当 中, 学生不叮就單独分出來受特殊訓練, 而应和一般数学專業一佯打好理論基礎, 然后

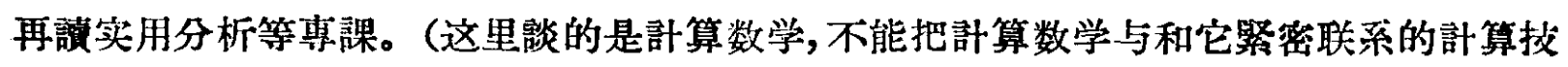
㭪研究混淆起來, 因为計算技術不單純是数学家的研究对像, 而应是数学家与工程技衔 專家等共同研究的对像。这点必須附帶說明。)

最后, 为了說明計算数学是数学整体的一个不可分割的部分, 它和数学的其他分支

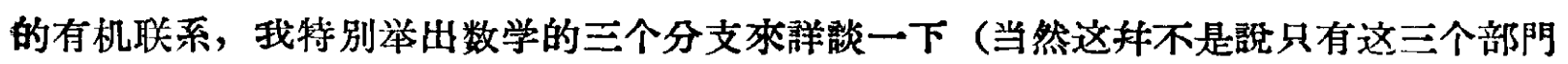
和計算数学有关)。

1. 計算数学与沉函分析。近代沉函分析为計算数学提供了許多新想法和新算法。如 果證在 20 世紀 30 年代, 当沉函分析开始以数学中一个具有独特对像与方法的分支出現 时, 不少数学家还对它的价值持保留的态度, 認为沉画分析似乎只能用統一的观点概括 数学分析中的已有成果, 似乎它不能用來發現新的事实, 那末, 在 20 年后的今天, 沉函分 析的重要作用应当对于数学家來說已是足够明顯的了。單就計算数学詮, 沉函分析在其 中的应用也已引起較大的注意。但是, 我們更应当注意沉函分析与計算数学的本貿关联。 实际上, 如苏联索伯列夫院士最近所指出〔見他在莫斯科罗蒙諾索夫大学 200 周年紀念 会（1955年 5 月11-13日）上的报告提綱, 刊載在苏联Успехи Матөматичөских Наук, $10: 3$ (1955), 194-195], 从現代数学的覌点來看, 計算数学的一切基本对像, 如近似 地表示一函数的圖、表、近似公式、在个別点处的数值等等, 都可以看作是在無窮維的 函数空間中的有蒻維近似。但如何研究不能变成有窮維的集呢? 这里度义分析已旦为这 一問題准备了基礎概念。所謂一定空間中某集 $\mathrm{E}$ 的一个有擳 $\varepsilon-$ 網, 是指由空問中有躬多 个点組成的集, 使 $\mathrm{E}$ 中每个点对这些有笨多个点之中的一个距离小于 $\varepsilon$ ( $\varepsilon$ 是桨正数)。 为了对于任意小的正数 $\varepsilon$, 集E恒有有籍 $\varepsilon$ - 網, 必須且只須这集 $\mathrm{E}$ 是列緊的。由此, 度义 分析中所提供的列緊性概念就成为計算数学的一切对像的重要屬性。近十年來沉函分析 在計算数学.上的应用方面已經有些有趣的絡果, 特別是苏联 Л.В. 坎托洛維赤敉授的学 派。在某些情形, 从沉画分析的一般观点不僅把不同方程的解法獲得統一处理, 而且其 所獲得的結果与佔值竟会比在个別問題中用特殊方法獲得的更精确。但这些还只是这一

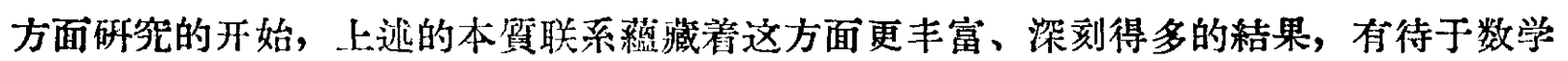
家的發掘。

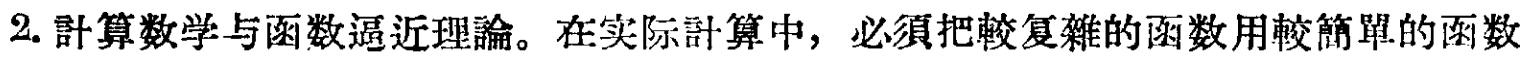
代替，例如用多項式代替。特別在数学計算机中，一划运算必須化成加、減、乘等运算， 而極限程序是一种無限程序, 不能在这种計算机上完全实現。因此与極限概念有着本婑

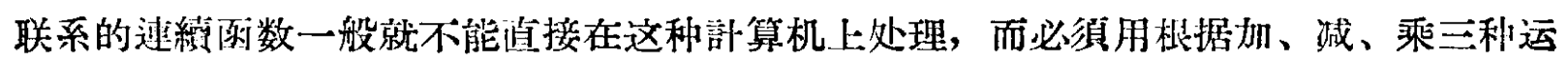
算而形成的多項式來近似地代替它。这种問題屬于函数逼近論。这一学科在其䋒造者 П.Л. 啠具等夫 $(1821-1894)$ 及苏联 C.H. 具尔恩史坦院士所预導的学派的手中已經在理 論上獲得了很好的發展。但在实际計算中求一已知連續函数的“最好逼近”种不是很容易 的事情, 我們还缺之一般的能行方法。与此相类似的还有插值理諭, 特別是多变数函数 的插值問題, 也还有待于進一步的研究。

3. 計算数学与数理邏輯。計算数学要求对各种数学問題找出獲得数值解的具体步驟, 
这事涉到数学中一个基本概念, 即算法(алгоритм)。所謂算法, 最近几年已獲得精确的 定义，粗略地證，是指一定的形式法則，这种法則使得某一类型的一邚闗題都能通过确 定的、自动的演算而解决。由此可以看出算法論与計算数学的本貿关系。由于快速計算 机的發展, 几乎任意理論上制定出來的算法都可以在实踐上实現, 反之, 某些算法論中 的否定性結果, 即对于某一类型的問題不存在一个一般的算法, 对于郚算数学如同样具 有重要意义。这一数学部門在数学中歷史也是較短的, 它有着無限的發展前途, 而色与

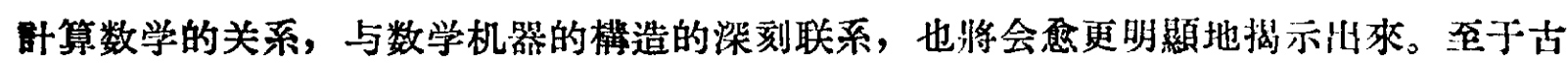
典命題演算在聯电器系統方面的应用还是比較初步的联系, 但这方面也还在吸引着很多 数学家的注意, 特別是人民民主國家中不少位数理䍜輯家最近在这方面有着不少工作。 在苏联科学院出版的数学評論雜誌上有 “电路的数学理論”这一專欄, 这一事实也起馀明 这方面研究的活躍情况。数理邏輯同計算数学、快速电子訪算机的本質联系也还表現在 其他一些方面, 有待于更好的發掘。

以上只是一些不系統的意見, 提出來供國內数学工作者参考。計算数学还是有着然 限發展前途的一阴学科, 也荤涉到很多数学根本問題, 希望更多的数学工作者注意它, 以便使得它作为整个数学的一个重要环篩健康地在我國發展起來。

\section{对發展半導体科学技術 研究的一些意見}

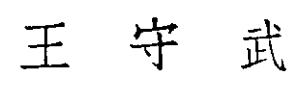

（中國科学院应用物理呼究所）

苏联科学院院長涅斯米掦諾夫院士在去年 12 月 31 日基理报上所發表的文章中指出， 苏联在發展牛導体方面所化的力量, 僅次于原子能和本利用方面。为什么苏联要用这样 大的力量棣發展牛導体科学技術呢? 我的体会是:

1. 几年來牛導体科学技術的笑飛猛進, 已經在無綫电工程、电力工程、自动控制和 國防工業上引起了巨大的革新。牛導体放大器已經可以代替大部分的电子管，牛導体整 流器已經被应用到几百几千乃至几万安培的大整流設备中去。牛導体的热电轉換器（發 比机）已經可以在農村中解决中小型功辣的电源問題。在利用太陽能的問題上，牛導体 器件也是不能被忽悓的。牛導体的光电池可以直接把太陽能轉換为电能, 其效率可以达 到 $10 \%$ 。此外，半導体的光敏电阻和热飭电阻都已被度泛地应用到自动控制和测量技 誧的各个方面去。这些牛導体器件的应用, 不僅是替代了原有的旧的器件, 而且有它們 